\title{
Binding Sites for Lewis Antigens Are Expressed by Human Colon Cancer Cells and Negatively Affect Their Migration
}

\author{
Axel Hittelet, Isabelle Camby, Nathalie Nagy, Hugues Legendre, Yves Bronckart, \\ Christine Decaestecker, Herbert Kaltner, Nikolay E. Nifant'ev, Nicolai V. Bovin, \\ Jean-Claude Pector, Isabelle Salmon, Hans-Joachim Gabius, Robert Kiss, and \\ Paul Yeaton
}

Departments of Gastroenterology (AH) and Pathology (NN, IS), Erasmus University Hospital, and Laboratory of Histopathology (IC, YB, CD, RK), Faculty of Medicine, Université Libre de Bruxelles, and Department of Surgery (HL, J-CP), Jules Bordet Institute, Brussels, Belgium; Institute of Physiological Chemistry (HK, H-JG), Faculty of Veterinary Medicine, Ludwig-Maximilians-University, Munich, Germany; Zelinsky Institute of Organic Chemistry (NEN) and Shemyakin Institute of Biorganic Chemistry (NVB), Russian Academy of Sciences, Moscow, Russia; and Digestive Health Center (PY), University of Virginia Health System, Charlottesville, Virginia

\begin{abstract}
SUMMARY: In colon cancer, endothelial cell selectins can promote tumor cell attachment via interactions with sialylated Lewis antigens present at the surface of tumor cells, thereby facilitating tumor cell arrest and transmigration into the extravascular space. However, it is not known whether Lewis antigens interact with colon tumor cells and modify their migration. Our aim was to detect the presence of binding sites on human tumor cells for Lewis ${ }^{a / x}$ antigens and their sialylated derivatives in vitro and in vivo and to analyze their influence on migration of colon cancer cells. The immunocytochemical and histochemical levels of expression of the four Lewis antigens were quantitatively determined in four human colon cancer cell lines and in in vivo nude mice xenografts. The levels of expression of specific binding sites for these sugar epitopes were determined by synthetic neoglycoconjugates. The influence of binding of these carbohydrate ligands on cancer cell migration was quantitatively evaluated by computer-assisted phase-contrast videomicroscopy performed on Matrigel culture supports either left uncoated or coated with neoglycoconjugate presenting synthetic Lewis ${ }^{a}$, sialyl Lewis ${ }^{a}$, Lewis ${ }^{\times}$, or sialyl Lewis ${ }^{\times}$antigens. The influence of the calcium concentration in the culture medium on the Lewis antigen-mediated effects was checked. Human colon cancer cells expressed significant amounts of specific binding sites detected by the synthetic probes in addition to the oligosaccharide epitopes. The expression levels differed considerably between the four cell lines and between in vitro and in vivo specimens. Cell migration analysis revealed that the four Lewis antigens markedly decreased the levels of migration of the HCT-15 and LoVo cancer cells. This effect depends on the calcium concentration in the culture medium. Binding sites for Lewis epitopes are present on colon cancer cells. The functional relevance of these sites is indicated by the negative influence on cell migration of a matrix containing the oligosaccharides as ligand parts. (Lab Invest 2003, 83:777-787).
\end{abstract}

$I$ $\mathrm{n}$ the majority of cases, the clinical end point of colon cancer is the destruction of the liver caused by the diffuse and total colonization of this organ by migrating colon cancer cells. As reported by Khatib et al (1999), the ability of disseminated cancer cells to

\section{DOI: 10.1097/01.LAB.0000073129.62433.39}

Received April 3, 2003.

IC is a Scientific Research Worker, CD is a Research Associate, and RK a Director of Research with the Fonds National de la Recherche Scientifique (Belgium). The present study was supported by grants awarded by the "Fonds de la Recherche Scientifique Médicale" (Belgium), "Les Amis de l'Institut Bordet" (Brussels, Belgium), the Dr.-M.-Scheel-Stiftung firr Krebsforschung (Bonn, Germany), and the W.-Sander-Stiftung (Munich, Germany).

Address reprint requests to: Dr. Robert Kiss, Laboratory of Histopathology, Faculty of Medicine, Université Libre de Bruxelles, 808 route de Lennik, cp620, 1070 Brussels, Belgium.E-mail: rkiss@ulb.ac.be establish metastases in secondary organs is regulated by a combination of factors including access to the organ microvasculature and specific host-tumor cell interactions. These authors also state that the attachment of circulating tumor cells to the vascular endothelium of the target organ is thought to be one of the key steps in the metastatic cascade. The available evidence suggests that this attachment both precedes and is required for tumor cell extravasation and the subsequent invasion of the target organ parenchyma. In search of biochemical epitopes that guide the biologic behavior, attention is increasingly directed to cell surface carbohydrates and their receptors, the endogenous lectins (Fukuda, 1995; Gabius, 1997, 2000; Listinsky et al, 1998; Muramatsu, 1993). Among them, the selectins belonging to the C-type family serve as a role model for lectins active in cell adhesion (Kansas, 1996; Tedder et al, 1995; Vestweber and 
Blanks, 1999). In contrast to most other adhesion molecules, selectin functions were initially thought to be restricted to leukocyte interaction with the vascular endothelium (Kaltner and Stierstorfer, 1998; Tedder et al, 1995; Vestweber and Blanks, 1999). However, several studies have demonstrated that selectins can act as homing receptors at the lumenal surface of the microvascular endothelium for tumor cells and that hematogenous metastasis of cancer involves recognition of tumor cell surface sugar epitopes, ie, sialylated Lewis epitopes, by selectins, thereby facilitating tumor cell arrest and transmigration into the extravascular space (Biancone et al, 1996; Brodt et al, 1997; Kaji et al, 1995; Kannagi, 1997; Nakamori et al, 1993; Tozeren et al, 1995).

Lewis antigens are commonly found at the termini of carbohydrate antennae with spatial accessibility for

A

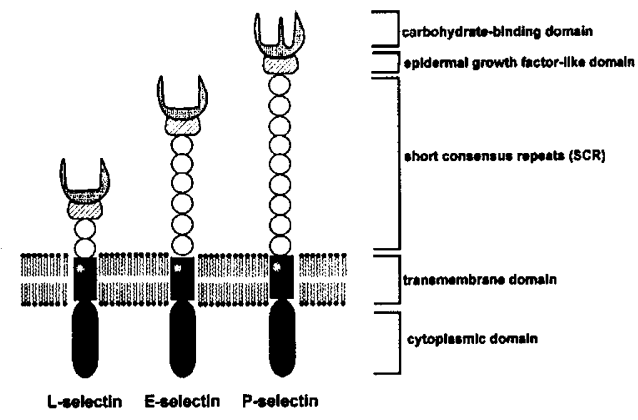

B

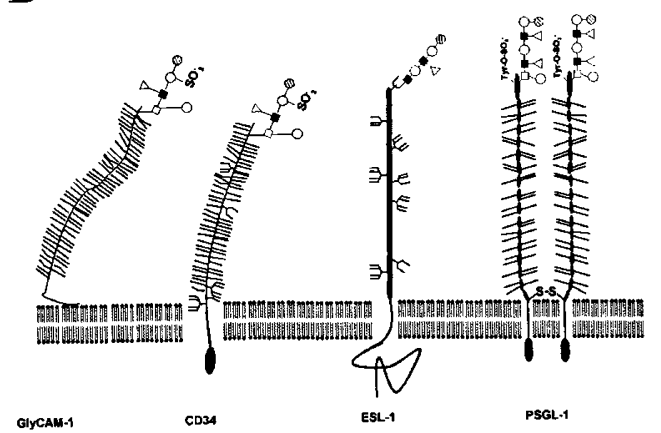

molecular recognition. Upon sialylation or sulfation, a crucial contact point for selectin interaction is established (Angata and Varki, 2002; Fukuda et al, 1999; Reuter and Gabius, 1999; Varki 1997; Vestweber and Blanks, 1999). Figure 1A details the modular structures of selectins, and Figure $1 \mathrm{~B}$ gives the schematic structures of known selectin-binding glycoproteins. Figure $1 \mathrm{C}$ illustrates the structure of the sialylated Lewis antigens and the biogenic relationship to further members of this family and the blood group antigens of the $\mathrm{ABH}$ family. Using studies of adhesion of tumor cells to vascular endothelium, with a focus on sialylated Lewis epitopes, it was noticed that the predominance of both the sialyl Lewis ${ }^{a}$ and Lewis ${ }^{x}$ isomers could vary with functional relevance for gastrointestinal tract tumors (Krause and Turner, 1999; Takada et al, 1991).

\section{$\mathrm{C}$}
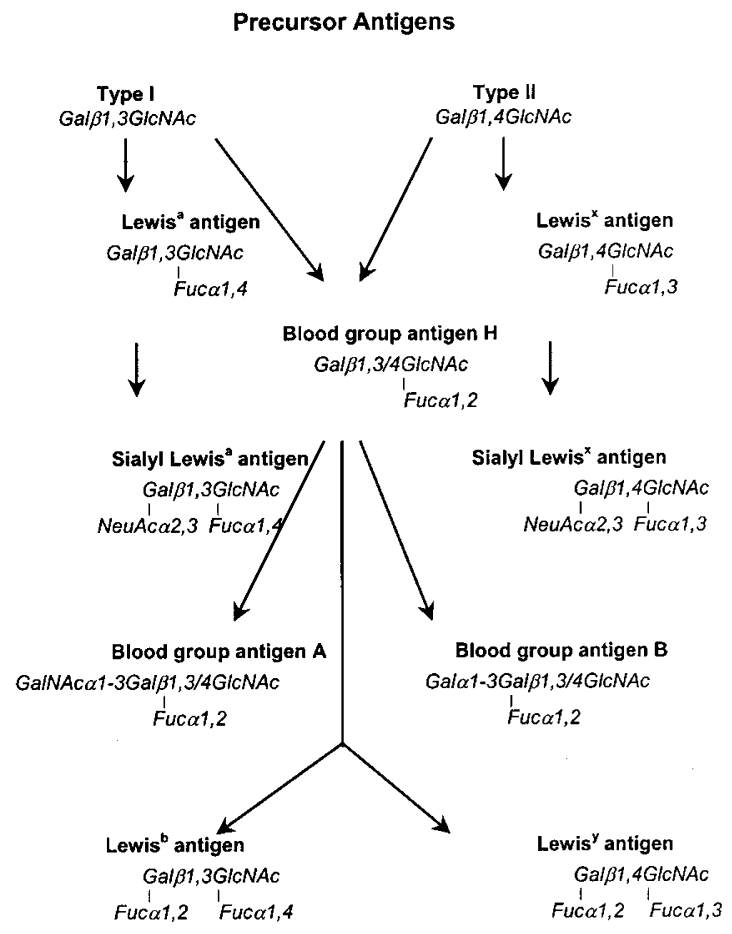

Figure 1.

Structural characteristics of selectins $(A)$ and some selectin ligands (B). The modular arrangement of the three members of the selectin family starts with an extracellular N-terminal lectin domain and an epidermal growth factor-like region (A). Two (L-selectin), six (E-selectin), and nine (P-selectin) consensus repeats homologous to a complement-binding domain, a transmembrane region, and a cytoplasmic C-terminal domain complete the modular arrangement of these C-type lectins (A). B, Schematic illustration of natural selectin ligands carrying the carbohydrate motifs outlined in $\mathrm{C}$. These carbohydrate moieties are crucial for binding to the selectin CRDs (A) and are presented by glycoproteins serving as selectin ligands (B). GlyCAM-1 or CD34, two heavily 0-glycosylated mucin-like proteins, are in vitro ligands of $L$ selectin. Both are expressed constitutively on the high endothelial venules of peripheral lymph nodes. Although GlyCAM-1 lacks a transmembrane domain and is most probably a secretory protein, CD34 is a type 1 transmembrane glycoprotein of 140 amino acids. ESL-1 is a ligand proposed for E selectin. In contrast to the mucin-type ligands with abundant glycosylation, this glycoprotein is sparsely decorated by $\mathrm{N}$ glycans. The $\mathrm{P}$ selectin glycoprotein ligand-1 (PSGL-1) is a disulfide bridge-linked dimeric type 1 transmembrane protein of 402 amino acids isolated from human leukocytes. The symbols used to describe the carbohydrate moieties located at the tips of the selectin ligands are as follows: open circles, D-galactose; open squares, N-acetyl-D-galactosamine (GalNAc); hatched circles, $\mathrm{N}$-acetyl-D-neuraminic acid (Sia); black squares, N-acetyl-D-glucosamine (GlcNAc); and open triangles, L-fucose (Fuc). The single spindles on the backbone of the protein carrier represent 0 -linked glycans, whereas the chains with branches represent $\mathrm{N}$-linked glycan antennae. The sizes of the individual parts of the scheme cannot be true to scale if the structural details of the crucial sites on the glycan part, their density, and their arrangement on the carrier and the backbone itself are to be illustrated in one figure (adapted from Kaltner and Stierstorfer, 1998). C, Schematic illustration of the biochemical pathways leading to the sialylated (or sulfated) Lewis antigen motifs that represent the active part of the ligands in the case of selectins $(A)$. Each arrow indicates the biochemical pathway leading from one sugar

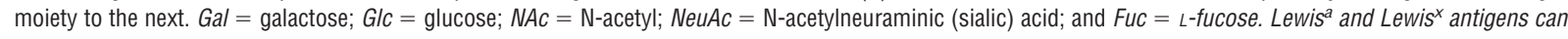
be synthesized when $\alpha$ 1,3-fucosyltransferase is present. In contrast, erythroid cells, which lack $\alpha 1,3$-fucosyltransferase, express $\alpha 1,2$-fucosyltransferase. Resultant $\mathrm{H}$-type oligosaccharides (O blood type) are converted into $A$ and $B$ blood group antigens by the addition of $\alpha 1,3$-linked N-acetylgalactosamine or galactose through the erythroid cell-specific expression of two distinct glycosyltransferases (Fukuda et al, 1999). 
Although it is therefore documented that host-cell selectins can promote tumor cell attachment to the endothelium via interactions with tumor-cell sialyl Lewis antigens, to our knowledge no study has ever reported evidence for the presence of binding sites for these sugar epitopes on colon cancer cells or the influence of Lewis antigens on the level of migration of these cancer cells. In fact, the sialylated derivatives of the Lewis epitopes are currently almost automatically linked to selectins as receptors. However, several lines of evidence, for example from patients with defects in fucose incorporation that cause severe symptoms beyond the immune system, challenge this almost paradigmatic view (Dell et al, 1999; Etzioni and Tonetti, 2000; Freeze, 2001; Ohyama et al, 1999; Yoshitani et al, 2001; Zhang et al, 2002), encouraging further studies. The aims of the present work were therefore to detect binding capacity and then, if positive, to characterize the influence of various types of Lewis antigens on the level of migration of these colon cancer cells. For this purpose, it is essential to synthesize the oligosaccharides and to link them to an inert carrier to mimic the high affinity-generating multivalency of their presentation in natural glycoconjugates, a route previously exploited for preparation of effective inhibitors for selectins and the galectin class of endogenous lectins (André et al, 1999, 2001; Gabius et al, 1993; Ohyama et al, 2002; Sanders et al, 1999; Simanek et al, 1998; Welply et al, 1994; Yamazaki et al, 2000). To exclude the possibility of exceptional behavior of a single line, we used four distinct cancer cell lines (HCT-15, LoVo, Colo-201, and DLD-1). They were cultured on plastic supports either left uncoated (control) or coated with a biologically inert polymer backbone carrying the synthetic Lewis $^{a}$, sialyl Lewis ${ }^{a}$, Lewis ${ }^{\times}$, or sialyl Lewis ${ }^{\times}$epitopes as potential effectors. The level of migration of living colon cancer cells was quantitatively determined by computer-assisted phase contrast videomicroscopy (De Hauwer et al, 1997, 1998). Because the conditions of cell growth had been reported to have a bearing on the extent of expression of a galactoside-binding lectin (Gabius and Vehmeyer, 1988), we included monitoring of xenograft material to rigorously define any influence of this parameter for these activities. Monitoring of cellular features included the mapping of expression of saccharide epitopes of the Le family and, as innovative step in this part of our work, of complementary endogenous binding sites. The level of expression of the four Lewis antigens in the four human cancer cell lines was quantitatively determined at the immunocytochemical level by computerassisted microscopy (Camby et al, 1999; Goldschmidt et al, 1996), as was done for the level of expression of the endogenous binding sites for these Lewis antigens using labeled neoglycoconjugates (Danguy et al, 1998; Gabius, 2001). We thus used these neoglycoconjugates to identify the status and modifications occurring at the level of carbohydrate receptors, a crucial step in the functional study of lectinomics. Because selectin binding to sialylated Lewis antigens, as with other C-type activities, is calcium dependent
(Reuter and Gabius, 1999; Varki, 1997), we also checked whether modification of the calcium concentration in the culture medium significantly modified the Lewis antigen-mediated influences on human colon cancer cell migration.

\section{Results}

\section{Quantitative Determination of the Immunocytochemical and Histochemical Expression of Lewis ${ }^{a}$, Sialyl Lewis ${ }^{a}$, Lewis $^{x}$, and Sialyl Lewis ${ }^{x}$ Antigens and of Specific Binding Sites for These Oligosaccharides in the HCT-15, LoVo, DLD-1, and Colo-201 Human Colon Cancer Cell Lines}

Although it could be expected that these cell lines are positive for the carbohydrate epitopes, we present the first evidence of the cells' capacity to bind these sugar ligands. Figure 2, A and B, illustrates the levels of expression of both classes of markers for cells obtained from in vitro cultures and from in vivo xenografts in terms of the percentages [determined by means of the labeling index (LI) variable] of colon cancer cells. Figure 2, C and D, illustrates the determined expression levels in terms of staining density [determined by the mean optical density (MOD) variable] per cell analyzed. Figure 2, A and C, shows the determined levels of expression of the Lewis antigenbinding sites, and Figure 2, B and D, represents the results of the determination of the levels of expression of the Lewis antigens themselves. The changes in the parameters of $\mathrm{LI}$ and MOD when testing the different oligosaccharides as parts of neoglycoconjugates, as seen in Figure 2, A and C, serve as further internal controls for specificity of the underlying recognition process.

We monitored cell material from culture or nude mice to determine any influence of the growth condition on these parameters. The percentages of HCT-15 $(p<0.01)$ and LoVo $(p<0.01)$ cells harboring Lewis ${ }^{a}$ antigen-binding sites were significantly lower in vivo than in vitro, whereas these percentages were similar $(p>0.05)$ in both the DLD-1 and the Colo-201 cells (Fig. 2A). The percentages of glycohistochemically positive cells in the case of the sialyl Lewis ${ }^{a}$ antigenbinding sites were significantly lower in vivo than in vitro for the HCT-15 ( $p<0.001)$, DLD-1 $(p<0.05)$, and Colo-201 $(p<0.01)$ cells, but not for the LoVo $(p>$ $0.05)$ cells. In the case of the Lewis ${ }^{\times}$antigen-binding sites, the percentages of glycohistochemically positive cells were significantly lower in vivo than in vitro for all four cell lines, ie, HCT-15 ( $p<0.001)$, LoVo ( $p<$ 0.01 ), DLD-1 ( $p<0.001$ ), and Colo-201 ( $p<0.05$ ) (Fig. $2 A)$. The same feature was observed for the sialyl Lewis $^{\mathrm{a}}$ antigen-binding sites in the HCT-15 ( $p<$ 0.001), DLD-1 ( $p<0.01)$, and Colo-201 ( $p<0.001)$ cells, but not in the LoVo ones $(p>0.05)$. These data indicate that the levels of expression of binding capacity for the four Lewis antigens investigated in the present study were globally down-regulated in vivo as compared with in vitro. A completely distinct pattern of expression was observed with respect to the in vitro 
A) Biotinylated Lewis Antigens

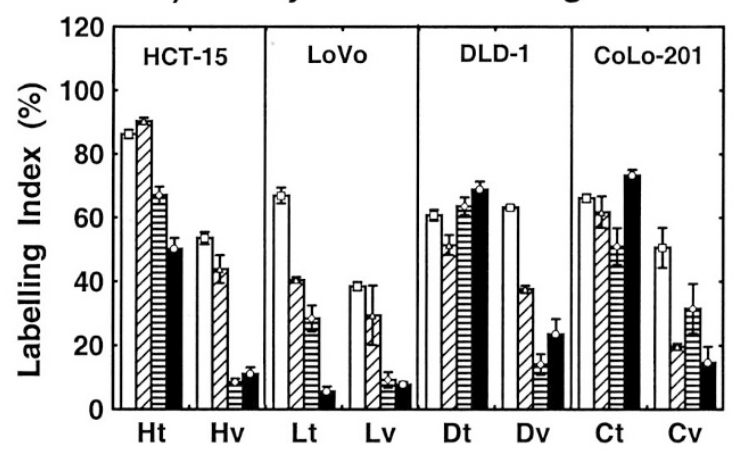

B) Anti-Lewis Antigen Antibodies

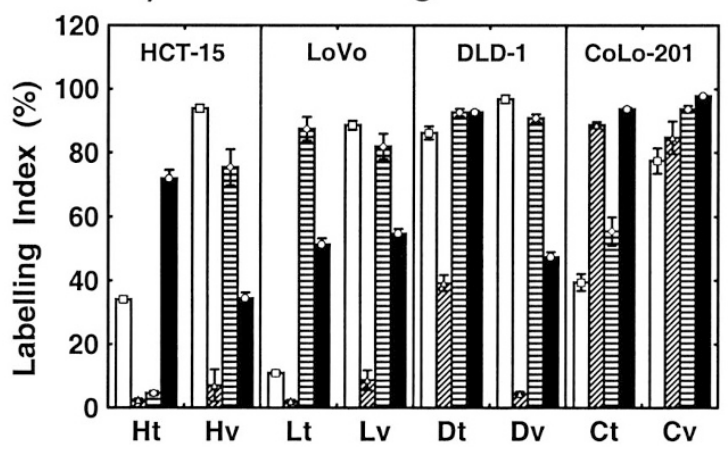

C) Biotinylated Lewis Antigens

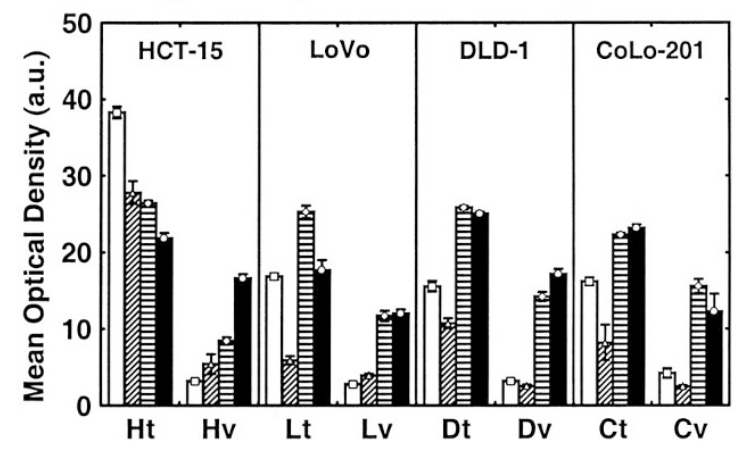

D) Anti-Lewis Antigen Antibodies

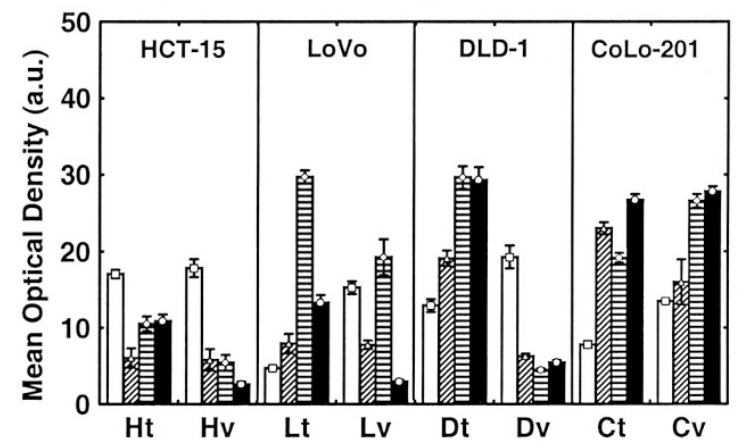

Figure 2.

Means \pm SEM with respect to the percentages (labeling indices) of human colon cancer cells expressing Lewis antigen-binding sites (A) and Lewis antigens themselves (B). The open bars represent data for the Lewis ${ }^{\mathrm{a}}$ antigen, the slant-hatched bars for the sialyl Lewis ${ }^{\mathrm{a}}$ antigen, the horizontal-hatched bars for the Lewis ${ }^{\mathrm{X}}$ antigen, and the solid bars for the sialyl Lewis ${ }^{x}$ antigen. $H=$ HCT-15; $L=$ LoVo; $D=$ DLD-1, $C=$ CoLo-201; $t=$ in vitro; $v=$ in vivo. Similar to A and B, panel $\mathrm{C}$ and $\mathrm{D}$ illustrate the levels of expression in terms of Lewis antigen-binding sites $(C)$ and Lewis antigen (D) density (determined by means of the mean optical density variable) per cell analyzed. The rest of the legend is identical to that for $A$ and $B$.

versus in vivo percentages of the human colon cancer cells for the four Lewis epitopes.

In fact, a marked increase in the percentage of immunohistochemically positive Lewis antigen cells was observed for the HCT-15 $(p<0.001)$, the LoVo ( $p$ $<0.001$ ), the Colo-201 ( $p<0.001$ ), and even for the DLD-1 $(p<0.05)$ cells when comparing the in vivo with the in vitro specimens (Fig. 2, B and D). Few of the HCT-15 and LoVo cells ( $<10 \%)$ were positive for the sialyl Lewis ${ }^{a}$ antigen. The DLD-1 cells showed less ( $p$ $<0.001$ ) immunohistochemical reaction for the sialyl Lewis $^{a}$ antigen in vivo than in vitro, whereas these percentages were both high $(>80 \%)$ and similar $(p>$ $0.05)$ in vitro and in vivo for the Colo-201 cells. The percentages of immunohistochemically positive cells for the Lewis ${ }^{\times}$antigen were significantly higher in vivo than in vitro for the HCT-15 $(\rho<0.001)$ and the Colo-201 $(p<0.01)$ cell lines, while remaining similar $(p>0.05)$ in the LoVo and DLD-1 lines. The percentages of cells expressing the sialyl Lewis ${ }^{\times}$antigen were significantly lower in vivo than in vitro for the HCT-15 $(p<0.001)$ and DLD-1 $(p<0.001)$ cells but remained similar $(p>0.05)$ for the LoVo and Colo-201 cells. These data strongly suggest that complex and probably multifactorial influences control the levels of expression of both Lewis antigens and their binding sites, because these levels differ markedly in vitro as compared with in vivo. In addition, none of the four Lewis antigens investigated in the present study emerged as a major determinant in the four cell lines analyzed. This means that any of the four Lewis antigens could be operative in recognitive interactions without preference given to a predominant determinant. Figure 2, C and D, shows that the density of each Lewis antigen per cell varied markedly from one cell line to another.

Because sialylated Lewis antigens interact with selectins, we also measured the levels of in vitro expression of E- and P-selectin in the four cell lines by means of quantitative immunofluorescence assays. Figure 3 shows that the different cell lines analyzed expressed these two selectins. Although higher levels of E-selectin were observed in the LoVo and the Colo-201 cells, $\mathrm{P}$-selectin seemed more uniformly expressed in the cell lines (except for a lower level in the Colo-201 cells).

\section{Characterization of the Influence of Neoglycoconjugates Presenting Lewis ${ }^{a}$, Sialyl Lewis ${ }^{a}$, Lewis ${ }^{x}$, and Sialyl Lewis ${ }^{x}$ Antigens on HCT-15 and LoVo Human Colon Cancer Cell Migration}

The data in Figure 2 show that binding sites for the four tested oligosaccharides are present on colon cancer cells, proving the validity of our initial hypoth- 


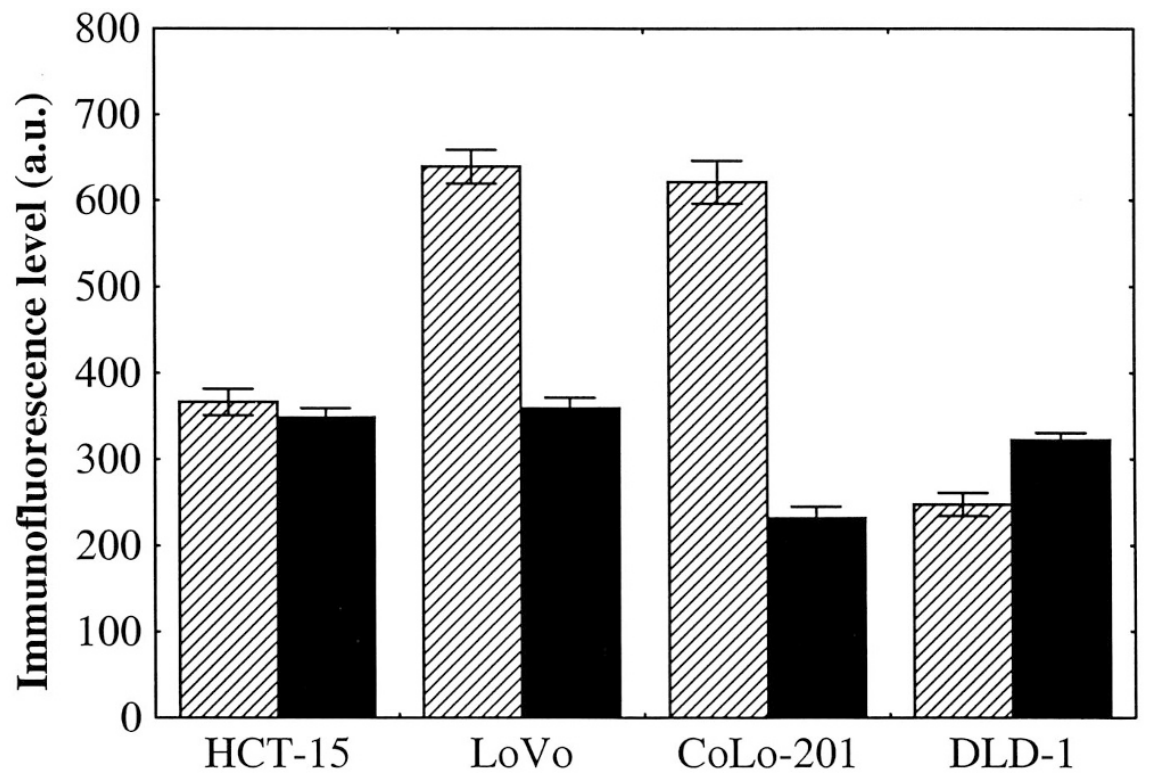

Figure 3.

Means \pm SEM with respect to the level of in vitro expression of E-selectin (hatched bars) and P-selectin (solid bars) revealed by means of immunofluorescence in the four human colon cancer cell lines analyzed in Figure 2.

esis. They also underline that the levels of expression of the four Lewis antigens and their binding sites markedly differed between the four cell lines analyzed. It is thus imperative to assay several lines for functional implications. The data detailed in Figure 4 reveal that, although the levels of the Lewis antigens and the Lewis antigen-binding sites were remarkably variable from one cell line to another, the presence of these sugar epitopes on the culture substratum consistently and markedly decreased the level of migration of these human colon cancer cells, at least in the HCT-15 and LoVo cell lines.

\section{Characterization of the Influence of the Calcium Concentration on the Effects of Sialyl Lewis ${ }^{x}$-Bearing Neoglycoconjugates on HCT-15 Colon Cancer Cell Migration}

Figure 4, C and D, reveals that, at least in the case of the tested HCT-15 cell line, effects of Lewis antigens, especially of the Lewis ${ }^{\times}$antigen, on human colon cancer cell migration are obviously dependent upon the calcium concentration, with a maximum effect reached between 1 and $5 \mathrm{~mm}$, when the threshold concentration is passed. Such calcium concentrations mediated effects to reduce HCT-15 colon cancer cell migration at the levels of migration speed [average cell speed (AS) variable in Fig. 4C] and the longest distance covered by the cells [maximum relative distance to the origin (MRDO) variable in Fig. 4D].

\section{Discussion}

Liver colonization by colon cancer cells can involve molecular interactions between selectins present on endothelial liver cells and sialylated Lewis antigens present on colon cancer cells. Whether lectin-Lewis antigen interactions are operative in colon cancer cell adhesion to endothelial liver cells only via selectins on the normal cells and whether a further type of interaction might then also affect colon cancer cell migration has not yet been clearly defined. As mentioned in the "Introduction," several lines of evidence support the notion that selectin-independent recognition of these carbohydrate epitopes can occur. Such a finding for colon tumor cells could definitely refine the way we view the functionality of these epitopes. We thus performed our study in two steps to address these issues. First, we used synthetic carbohydrate ligands as part of a set of neoglycoconjugates to answer the pertinent question of whether tumor cells themselves have the capacity to bind these sugar epitopes. As detailed in the "Introduction," the chemical conjugation of the oligosaccharides results in affinity enhancement relative to the monovalent structure, mimicking its clustered presentation on natural glycoconjugates. Next, we used quantitative videomicroscopy to investigate whether lectin-Lewis antigen interactions might modify colon cancer cell migration. Experimental studies from the literature show that tumor cells colonizing the liver activate several cell types present in the liver (Kupffer cells, endothelial cells, circulating polymorphonuclear cells in the liver) to release various cytokines (including for example TNF- $\alpha$ ) which, in turn, up-regulate E-selectin expression (Khatib et al, 1999). Kobayashi et al (2000) showed that the level of E-selectin expression is a key event for liver colonization by tumor cells. Indeed, these authors demonstrated that tumor cell attachment to endothelial cells may be noticeably decreased by cimetidine, a histamine type 2 receptor antagonist, which down-regulates cell surface expression of E-selectin on endothelial cells. In addition to E-selectin, tumor cells can also cause an increase in the expression of hepatic P-selectin and, subsequently, of intercellular adhesion molecule-1 
HCT-15 Cell Line

A) HCT-15 Cell Line MRDO Variable

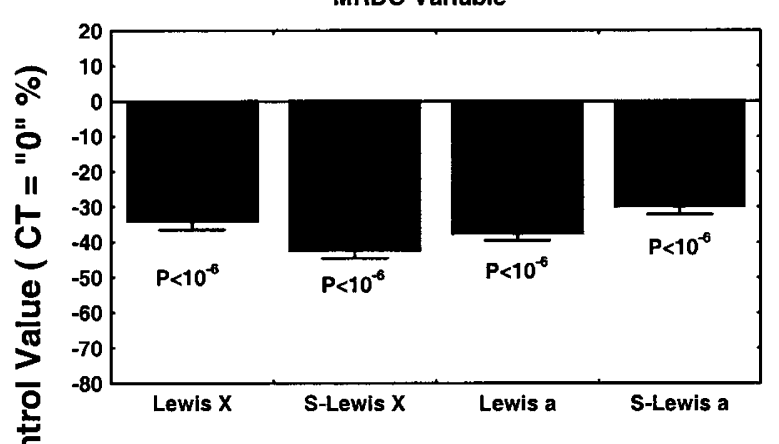

B) LoVo Cell Line MRDO Variable

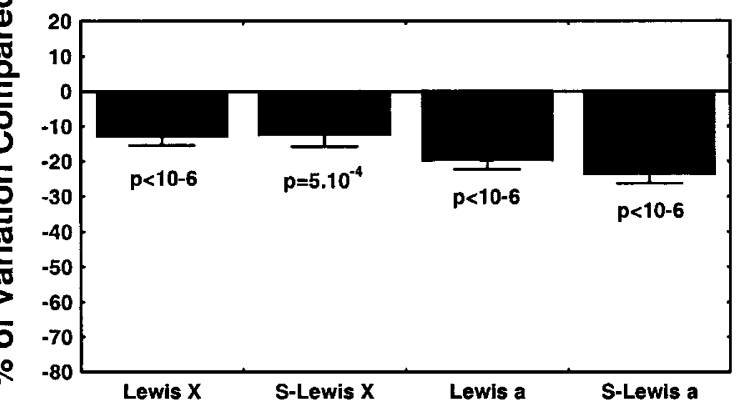

Cultured on Matrigel + Sialyl-Lewis X Antigen $\left(0.15 \mu \mathrm{g} / \mathrm{cm}^{2}\right)$

C) AS Variable

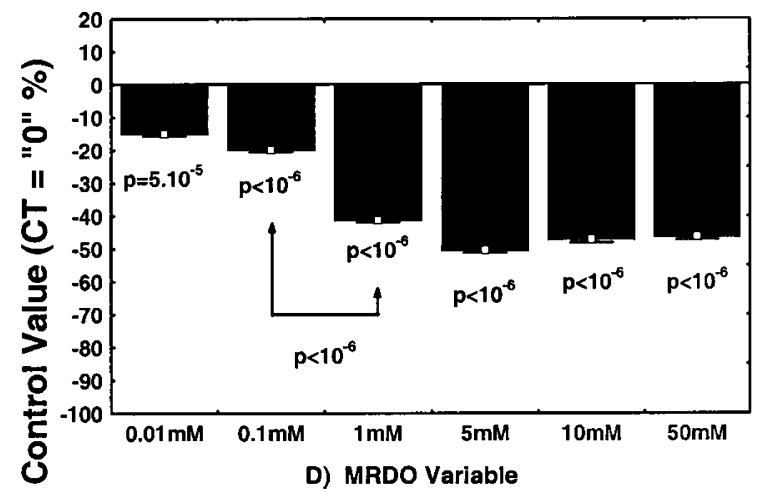

\section{Figure 4.}

The effects on the migration levels of vital HCT-15 (A) and LoVo (B) cells of Lewis ${ }^{\mathrm{x}}$, sialyl Lewis ${ }^{\mathrm{x}}$, Lewis ${ }^{\mathrm{a}}$, and sialyl Lewis ${ }^{\mathrm{a}}$ antigens coated $\left(\right.$ at $0.15 \mu \mathrm{g} / \mathrm{ml} . \mathrm{cm}^{2}$ ) on cell culture supports precoated with Matrigel when compared with the Matrigel-coated control condition. These effects are illustrated by means of the variations (means \pm SEM) observed with respect to control (control mean set to 0 ) in terms of the greatest linear distances covered (MRD0) by cells. $C$ and $D$, Influence of calcium concentrations (in the culture media) on the effects of the Lewis ${ }^{x}$ antigen on human colon cancer HCT-15 cell migration. While the $x$-axis indicates the different calcium concentrations investigated, the $y$-axis reports the variations (means \pm SEM) observed with respect to control (control mean set to 0 ) in terms of the average cell speed (AS, at the top) and the greatest linear distances covered (MRDO, at the bottom).

and vascular cell adhesion molecule-1 (Khatib et al, 1999).

The common view of the data in the literature is that colon cancer cells expressing sialyl Lewis antigens can readily attach to endothelial cells expressing Eand P-selectin. Sialyl Lewis ${ }^{\times}$work with B16 and MeWo melanoma cell clones after transfection with $\alpha 1,3-$ fucosyltransferase cDNA pointed to a dual role favoring metastasis at a low cell surface density and also natural killer (NK) cell attack above a certain threshold, CD94 being a likely receptor on the NK cells (Ohyama et al, 1999, 2002). Evidently, this oligosaccharide, the product of a complex enzymatic machinery (Fig. 1C), is an important docking point in cell communication. Our study explores a hidden aspect of its functionality. In the present work, we monitored colon tumor binding capacity for these sugar epitopes in detail. This is an entirely new aspect. However, we deliberately did not use a biotinylated oligosaccharide for this purpose. Indeed, the low affinity of the monovalent sugar and the lack of similarity to the natural way of glycoepitope presentation argue against this option, as already outlined in the "Introduction." In contrast, we exploited chemically designed neoglycoconjugates for this purpose, the first time such a sialyl Lewis ${ }^{x}$ decorated synthetic carrier has been introduced to a study in tumor biology.

The data from the present study clearly indicate that human colon cancer cells express significant amounts of binding sites for Lewis antigens (Fig. 2). Structuredependent alterations of staining parameters underscore the inherent specificity of the recognition process. We deliberately monitored four cell lines to avoid being led astray by a single case. In a further step, the molecular characteristics of these detected sites will be defined. The presented indication for $\mathrm{Ca}^{2+}$ sensitivity (Fig. 4) is strongly evocative of the behavior of C-type lectins. The fact that human colon cancer cells express both Lewis antigens and their ligands (Fig. 2) intimates that the presence of complementary binding partners could indeed favor clustering of colon cancer cells by trans-interactions. The data presented in Figure 2 also strongly suggest that the level of expression of both the Lewis antigens and the Lewis antigenbinding sites in human colon cancer cells are regulated by multiple factors, because these levels differ markedly from one cell line to another and between in vitro and in vivo specimens. 
The three selectins known today recognize sialyl Lewis $^{\times}$as a major determinant (Foxall et al, 1992; Tedder et al, 1995; Vestweber and Blanks, 1999). In the biologic behavior of colon cancer cells, the sialyl Lewis $^{\mathrm{a}}$ antigen was initially thought to play a more important role than the sialyl Lewis ${ }^{\times}$antigen (Takada et al, 1991). Prognostic evaluations point to a functional impact of both determinants, and an increased expression of sialyl Lewis ${ }^{x}$ correlated with the extent of malignancy and the high incidence of recurrence (and consequently with survival) of colon carcinoma patients (Nakamori et al, 1993). However, recent studies disagree with this conclusion, pinpointing a correlation of sialyl Lewis ${ }^{\times}$(but not the Lewis ${ }^{a}$ isomer) expression with progression of lymph node metastasis and dedifferentiation but not its status as an independent prognostic factor in colorectal adenocarcinomas (Baldus et al, 2002; Matsumoto et al, 2002). An important point to be noted for comparison is the nature of the $\mathrm{mAb}$ used as fine-specificity differences are encountered between various preparations (Matsumoto et al, 2002). At any rate, these clinical studies provide reason to pursue a delineation of functions of these oligosaccharides in models. Using a cell biologic approach to give a further example, Weston et al (1999) demonstrated that blocking the biosynthesis of sialyl Lewis $^{a}$ and sialyl Lewis ${ }^{\times}$epitopes by inhibition of $\alpha(1,3)$ fucosyltransferase expression prevented stable transfectant clones (clones of 29 LMM cancer cells transfected with antisense sequences directed against this enzyme's mRNA) from colonizing the liver (the parental 29 LMM cancer cells are highly metastatic to the liver). Consequently, thorough monitoring of Le epitope presence is advised in human colon cancer cell characterization. The quantitative determination of the level of expression of the Lewis ${ }^{a}$, sialyl Lewis ${ }^{a}$, Lewis $^{x}$, and the sialyl Lewis ${ }^{x}$ antigens revealed that the four cell lines that we used expressed Lewis antigens, notably in markedly variable amounts, and there were also major differences depending on whether these levels of expression were determined using in vitro or in vivo specimens (Fig. 2). Interestingly, in this context and as already mentioned above, Ohyama et al $(1999,2002)$ detected the potential of a dual role of the sialyl Lewis ${ }^{\mathrm{x}}$ epitope on metastasis and NK cell attack. In their studies these authors used stably transfected cell variants with $\alpha 1,3-$ fucosyltransferase cDNA and focused investigations on clones with variant glycoepitope density. In our study, we determined the level of this epitope as occurring in the cells and the tumors without genetic manipulation. In addition to seeing changes in this aspect for the different cells in vitro and their tumors in nude mice (without determining the implications on NK cell attack, which was not the focus of our study), we devoted special attention to the detection of binding sites for sialyl Lewis oligosaccharides and their sialic acid-free parental forms in the tumor cells as well as to the ensuing implications for tumor cell migration.

To characterize a functional impact for the newly detected presence of binding sites, we analyzed cell migration in the presence of custom-made neoglycoconjugates. Notably, an effect will serve as independent evidence for the cells' capacity to bind these epitopes. The quantitative determination of the influence of Lewis ${ }^{a}$, sialyl Lewis ${ }^{a}$, Lewis ${ }^{x}$, and sialyl Lewis ${ }^{x}$ on HCT-15 and LoVo cancer cell migration revealed that these four oligosaccharide determinants, as part of the culture substratum, markedly decreased the levels of this migration (Fig. 4). These data therefore point to a negative influence on cell migration of this type of protein-carbohydrate interaction.

In conclusion, this study applied a set of labeled neoglycoconjugates and determined by chemical synthesis and quantitative histochemistry that colon tumor cells express binding sites for Lewis epitopes along with the carbohydrate determinants. Expression levels are dependent on the type of cell line and on culture conditions. In addition to the detection of this cell characteristic, we performed an analysis of a cell property important for tumor progression, ie, cell migration. As a first functional parameter, we demonstrated a negative influence of the described binding capacity on cell migration. Further studies are in progress to evaluate the prognostic relevance of this new cell feature of colon cancer cells. Considering the approach to design synthetic inhibitors of selectincarbohydrate interactions (Simanek et al, 1998), the detection of selectin-independent recognition processes in colon cancer cells deserves attention to gauge target specificity.

\section{Materials and Methods}

\section{Cell Lines, Culture Media, and Nude Mice Grafting Procedure}

The four human colon cancer cell lines were obtained from the American Type Culture Collection (ATCC, Manassas, Virginia). These four lines included the HCT-15 (ATCC code CCL225), the LoVo (ATCC code CCL229), the CoLo201 (ATCC code CCL224), and the DLD-1 (ATCC code CCL221) models.

The cells were cultured at $37^{\circ} \mathrm{C}$ in sealed (air tight) Falcon plastic dishes (Nunc, Gibco, Belgium) containing Eagle's MEM (Gibco), supplemented with 10\% FCS. All of the media were supplemented with a mixture of $0.6 \mathrm{mg} / \mathrm{ml} \mathrm{L-glutamine} \mathrm{(Gibco),} 200 \mathrm{lU} / \mathrm{ml}$ penicillin (Gibco), $200 \mathrm{IU} / \mathrm{ml}$ streptomycin (Gibco), and $0.1 \mathrm{mg} / \mathrm{ml}$ gentamicin (Gibco). The FCS was heat inactivated for 1 hour at $56^{\circ} \mathrm{C}$.

In vivo nude mice xenografts were obtained from the HCT-15, LoVo, CoLo201, and DLD-1 in vitro cell lines by means of the procedure that we have described previously with respect to the grafting of human colon cancers onto this type of nude mouse (Jannot et al, 1993). Briefly, one million cells from each of the four cell lines were subcutaneously grafted onto 8-week-old female nu/nu mice (21-23 g; Iffa Credo, IFFA Credo, Arbresle, France). Five nude mice were grafted for each of the four cell lines. At the 28th day after grafting, the mice were killed and the tumors removed. Each tumor was fixed for 4 days in buffered 
formalin (4\%), dehydrated, and embedded in paraffin wax.

\section{Histochemical Procedures for Revealing the Presence of Lewis Antigens and Lewis Antigen-Binding Sites}

When working with the cell lines, we systematically used histochemical procedures to avoid any of the problems of membrane permeabilization likely to occur within cytochemical procedures. Thus, in the case of the four in vitro cultured cell lines, we obtained cell pellets by centrifuging 10 million cells from each of the four lines for 10 minutes at $800 \times g$. These pellets were then fixed for 4 hours in buffered formalin (4\%), dehydrated, and embedded in paraffin wax. Three pellets were available for each of the four cell lines. The histochemical procedures were performed as detailed previously (Camby et al, 1999; Delbrouck et al, 2002; Goldschmidt et al, 1996). Briefly, 5- $\mu$ m-thick sections were taken from each cell line pellet or xenograft sample. Incubation with the various antibodies or synthetic probes was performed at $25 \pm$ $1^{\circ} \mathrm{C}$ for 60 minutes under conditions involving minimal background staining and at the dilutions described below for each antibody. The extent of specifically bound antibodies or probes was visualized by avidinbiotin-peroxidase complex kit reagents (Vector Labs, Burlingame, California), with diaminobenzidine $/ \mathrm{H}_{2} \mathrm{O}_{2}$ as the chromogenic substrates. The control reactions for each of the four anti-Lewis antibodies under study included the omission of the incubation step in the case of the primary antibody and also the preincubation of the antibody with the antigen. The specificity of each antibody was checked by Western blotting (data not shown). The neoglycoconjugates were synthesized and biotinylated as described in detail elsewhere (Bovin and Gabius, 1995; Gabius and Bardosi, 1991; Nifant'ev et al, 1994, 1996). The control reactions for the application of the biotinylated probes included competitive inhibitions to ascertain sugar specificity; the omission of the incubation step with a labeled marker served to exclude any staining by the binding of kit reagents such as the mannose-rich glycoproteins horseradish peroxidase and avidin. Counterstaining was performed with hematoxylin. The origins and dilutions for each anti-Lewis antigen antibody were as follows.

(1) anti-Le $e^{x}$ (CD15): the anti-Le $e^{x}$ Ab was generated from the spleen cells of a BALB/c mouse that had been immunized with Salmonella minnesota coated with Lex-active glycolipids. This mAb specifically recognizes the $\mathrm{Le}^{\mathrm{x}}$ antigen (clone 73-30; Seikagaku Corporation, Tokyo, Japan; dilution $1: 100)$.

(2) anti-sLe $e^{x}$ the anti-sLe $e^{x} m A b$ was secreted by a hybridoma formed from the fusion of P3/X63-Ag8 mouse myeloma cells with spleen cells taken from a BALB/c mouse immunized against the human lung adenocarcinoma-crude membrane fraction. This antibody has been shown to recognize the sialylated carbohydrate epitope $\left(s L e^{x}\right)$ on antigens expressed in lung adenocarcinoma cells (clone KM-93; Seikagaku Corporation; dilution 1:100).

(3) anti-Le $e^{a}$ : the anti-Le ${ }^{a}$ mAb was obtained from a $B A L B / c$ mouse that had been immunized with mucin isolated from an ovarian cyst fluid, and the splenocytes were fused with mouse myeloma SP2/0 cells (clone 7LE; Seikagaku Corporation; dilution 1:100).

(4) anti-sLe $e^{a}$ : the anti-sLe $e^{a} m A b$ was obtained from the spleen cells of a BALB/c mouse immunized with human colon cancer cells (the CoLo201 cell line). This antibody preferentially recognizes the $2 \rightarrow 3$ sLe $^{a}$ antigen (Fig. 2) but also reacts against other structurally related sialylated type I chain carbohydrate antigens (Fig. 1C) (code 2D3; Seikagaku Corporation; dilution 1:100.

\section{Computer-Assisted Microscopy for Quantitative Histochemistry}

For each marker (ie, a biotinylated neoglycoconjugate or an antibody), two variables were processed by means of a SAMBA 2005 computer-assisted microscope system (Samba Technologies, Grenoble, France) with a $\times 20$ (aperture 0.50 ) magnification lens. $\mathrm{LI}$ refers to the percentage of tissue area specifically stained by a histochemical probe. MOD denotes staining intensity. The way in which we used the computerassisted system to quantify the histochemical staining is detailed elsewhere (Camby et al, 1999; Delbrouck et al, 2002; Goldschmidt et al, 1996). The computerassisted microscope and related quantitative analyses were standardized as follows. A negative histologic control slide (which was not exposed to either the biotinylated neoglycoconjugate or the primary antibody) was analyzed for each of the probes mentioned above and for each type of biologic sample under study, ie, the cell line pellets and the xenografts. The software used on the computer-assisted microscope automatically subtracted the $\mathrm{LI}$ and MOD values of the negative control sample from each corresponding positive one. Special software was used on the computer to assist the microscope in checking any inherent shading in the charge-coupled device (CCD) camera-based systems, the glare phenomenon, and the level of linearity precision. The shading and glare were checked each week. The monitoring performed by our computer-assisted microscope showed that neither the shading, the glare, nor the linearity significantly modified our results (data not shown).

Ten areas of $120,000 \mu \mathrm{m}^{2}$ were scanned for each of the biologic specimens under study (ie, the triplicate in vitro pellets and the five in vivo xenografts available for each cell line) and each marker was investigated here. Only the epithelial tissue was analyzed, and a computer mouse linked to the software on the computerassisted microscope enabled all of the nonepithelial tissue to be removed from the video control before digitizing of the histologic field to be analyzed. 


\section{Immunofluorescence Assays}

Immunofluorescent stainings were performed to confirm the expression of $E$ and $P$ selectin according to the procedure detailed elsewhere (Nagy et al, 2001). We used a polyclonal goat anti-human E-selectin antibody (1:200) and a monoclonal mouse anti-human P-selectin antibody (1:100; R\&D Systems Europe Ltd., Abingdon, United Kingdom). They were revealed by Alexa 594 (red) conjugated anti-goat or anti-mouse IgG (1:400; Molecular Probes, Eugene, Oregon).

In the course of each assay, photographs of between 50 and 100 cells per experimental condition (pooled from three independent experiments) were made with a $\times 100$ lens under the same carefully checked exposure conditions. The fluorescent signals were quantified with a PROVIS Olympus Microscope (Omnilabo S.A., Antwerp, Belgium) coupled to a Mega-view 2 camera (Omnilabo S.A.) feeding digitized information to a computer using an AnalySISt software (Soft Imaging System; GMbH, Munster, Germany).

\section{Quantitative Determination of In Vitro Cell Motility}

In comparison to the Matrigel-coated control condition, the influence on the migration levels of the living HCT-15 and LoVo cells of neoglycoconjugates presenting Lewis ${ }^{\times}$, sialyl Lewis ${ }^{\times}$, Lewis ${ }^{a}$, and sialyl Lewis ${ }^{a}$ antigens (each coated at $0.15 \mu \mathrm{g} / \mathrm{ml} . \mathrm{cm}^{2}$ on cell culture support precoated with Matrigel; Becton Dickinson, Meylan, France) was quantitatively determined by a computer-assisted phase-contrast microscope (Olympus, Antwerp, Belgium) equipped with a CCD camera (Hitachi Denshi, Japan). The technical procedure is described in detail elsewhere (De Hauwer et al, 1997, 1998; Delbrouck et al, 2002). Briefly, the software that we set up enabled each HCT-15 or LoVo cell in the colony under study (Fig. 5A) to be distinguished automatically on the basis of distinct morphologic characteristics that, in turn, enabled the cells to be identified against the background (in the present case, the plastic Falcon dishes) (Fig. 5B). Thus, the automatic segmentation of a cell from its background was followed by a process that enabled the coordinates of its center of gravity (centroid) to be computed (Fig. $5 \mathrm{C})$. Because this operation was performed every 4 minutes, the trajectory of each cell centroid could be computed by interpolation (Fig. 5D). Two motility descriptors were then determined from these trajectories for each human colon cancer cell subjected to the computer-assisted videomicroscopy procedure. The first descriptor was the average cell speed (the quantitative AS variable) (Fig. 5E). The second consisted of the maximum relative distance to the origin (the quantitative MRDO variable) of the cells (Fig. 5E). This latter variable was the greatest linear distance found between the original and subsequent positions of the cell
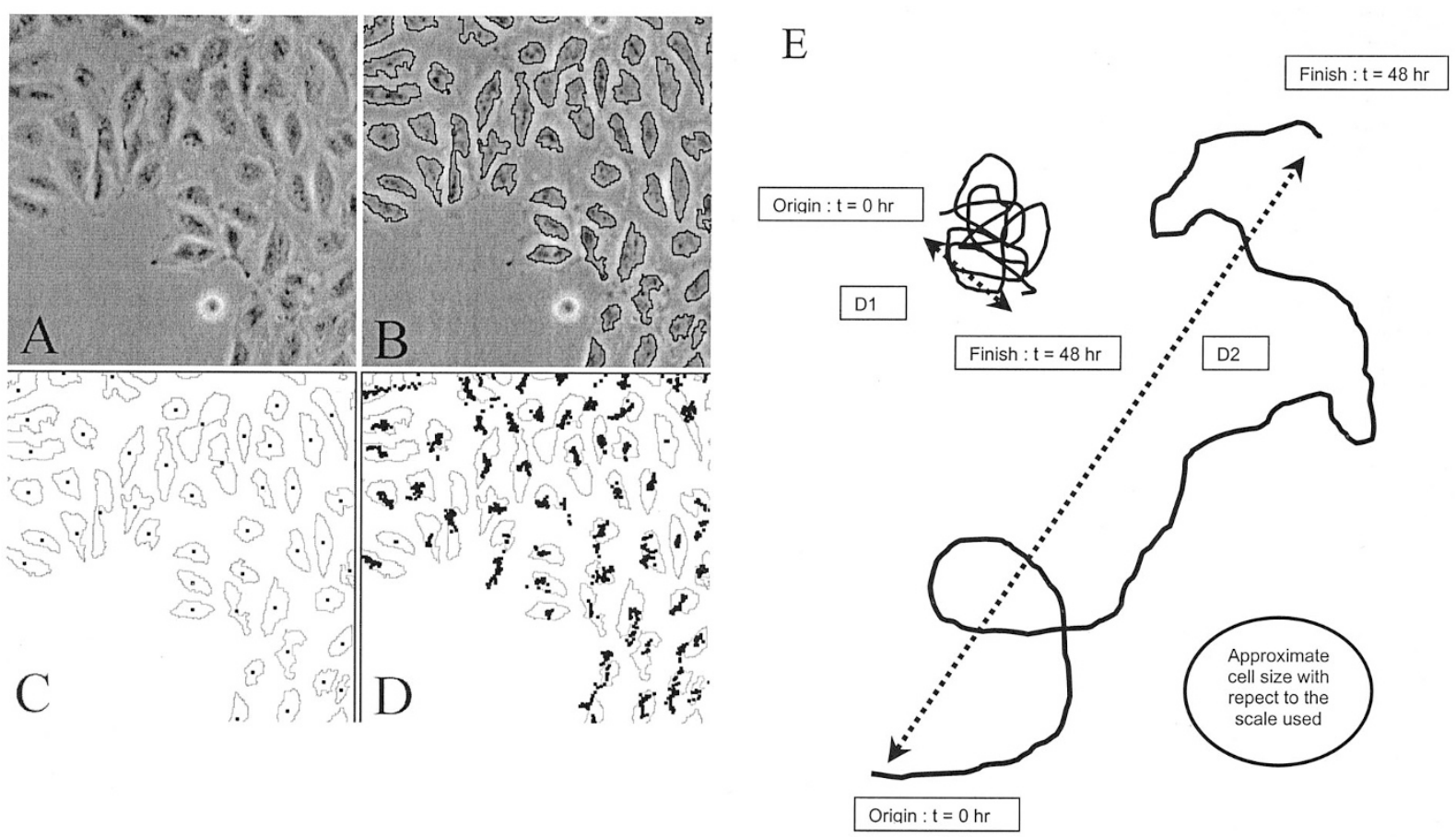

Figure 5.

Automatic cell image analysis performed by the software associated with our cell tracking system. A digital image of the microscopic field analyzed was taken every 4 minutes (A). Each cell was first identified against the background (B). For each cell so identified, the system computed the coordinates of its center of gravity (C). $D$, shows the results of this process applied to a series of digital pictures (taken every 4 minutes) enabling the trajectory of each cell centroid to be computed by interpolation. E, Schematic illustration of the trajectories of two cells with different types of behavior. Although the first one moves randomly and remains in a small area, the second follows a more extended trajectory. For each cell trajectory, the system computes two parameters to distinguish between the different kinds of cell behavior that can be encountered. The first one is the average speed (the AS variable) defined by the length of the cell trajectory divided by the time taken by the cell to cover it. The second is the maximum relative distance to the origin (the MRDO variable) which is defined by the greatest linear distance found between the original $(t=0)$ and any subsequent position of the cell (distances D1 and D2 in the figure), divided by the duration of the cell observation. 
divided by the duration of observation (De Hauwer et al, 1997, 1998; Fig. 5E). At the beginning of the experiment ( $\mathrm{t}=0 \mathrm{hr}$ ), between 25 and 45 malignant colon cells were present in the field of the phasecontrast microscope and were thus computed by the CCD camera. At the end of the experiment $(t=48 \mathrm{hr})$, the trajectories of between 260 and 410 malignant cells had been analyzed in each experimental condition; these analyses were performed in triplicate.

\section{Statistical Analyses}

The statistical comparisons of the data were performed by the Fisher $F$ test (one-way analysis of variance for more than two groups) or the Student's $t$ test (for two groups) after a check of the equality of variance by means of the Levene test and of the normal distribution fitting of the data by means of the $\chi^{2}$ test. When these parametric conditions were not satisfied, nonparametric Kruskall-Wallis (for more than two groups) or Mann-Whitney (for two groups) tests were performed. All statistical analyses were made using the Statistica software package (Statsoft, Tulsa, Oklahoma).

\section{References}

André S, Cejas Ortega PJ, Alamino Perez M, Roy R, and Gabius HJ (1999). Lactose-containing starburst dendrimers: Influence of dendrimer generation and binding-site orientation of receptors (plant/animal lectins and immunoglobulins) on binding properties. Glycobiology 9:1253-1261.

André S, Pieters RJ, Vrasidas I, Kaltner H, Kuwabara I, Liu FT, Liskamp RMJ, and Gabius HJ (2001). Wedgelike glycodendrimers as inhibitors of binding of mammalian galectins to glycoproteins, lactose maxiclusters and cell surface glycoconjugates. Chembiochem 2:822-830.

Angata T and Varki A (2002). Chemical diversity in the sialic acids and related $\alpha$-keto acids: An evolutionary perspective. Chem Rev 102:439-469.

Baldus SE, Monig SP, Hanisch FG, Zirbes TK, Flucke U, Oelert S, Zilkens G, Madejczik B, Thiele J, Schneider PM, Holscher AH, and Dienes HP (2002). Comparative evaluation of the prognostic value of MUC1, MUC2, sialyl-Lewis(a) and sialyl-Lewis $(\mathrm{x})$ antigens in colorectal adenocarcinoma. Histopathology 40:440-449.

Biancone L, Araki M, Araki K, Vassalli P, and Stamenkovic I (1996). Redirection of tumor metastasis by expression of E-selectin in vivo. J Exp Med 183:581-587.

Bovin NV and Gabius HJ (1995). Polymer-immobilized carbohydrate ligands: Versatile chemical tools for biochemistry and medical sciences. Chem Soc Rev 24:413-421.

Brodt P, Fallavollita L, Bresalier RS, Meterissian S, Norton CR, and Wolitzky BA (1997). Liver endothelial E-selectin mediates carcinoma cell adhesion and promotes liver metastasis. Int J Cancer 71:612-619.

Camby I, Nagy N, Lopes MB, Schafer BW, Maurage CA, Ruchoux MM, Murmann P, Pochet R, Heizmann CW, Brotchi J, Salmon I, Kiss R, and Decaestecker C (1999). Supratentorial pilocytic astrocytomas, astrocytomas, anaplastic astrocytomas and glioblastomas are characterized by a differential expression of S100 proteins. Brain Pathol 9:1-19.
Danguy A, Decaestecker C, Genten F, Salmon I, and Kiss R (1998). Application of lectins and neoglycoconjugates in histology and pathology. Acta Anat 161:206-218.

De Hauwer C, Camby I, Darro F, Decaestecker C, Gras T, Salmon I, Kiss R, and Van Ham P (1997). Dynamic characterization of glioblastoma cell motility. Biochem Biophys Res Commun 232:267-272.

De Hauwer C, Camby I, Darro F, Migeotte I, Decaestecker C, Verbeek C, Danguy A, Pasteels JL, Brotchi J, Salmon I, Van Ham P, and Kiss R (1998). Gastrin inhibits motility, decreases cell death and increases proliferation in human glioblastoma cell lines. J Neurobiol 37:373-382.

Delbrouck C, Doyen I, Belot N, Decaestecker C, Ghanooni R, de Lavareille A, Kaltner H, Choufani G, Danguy A, Vandenhoven G, Gabius HJ, Hassid S, and Kiss R (2002). Galectin-1 is overexpressed in nasal polyps under budesonide and inhibits eosinophil migration. Lab Invest 82:147-158.

Dell A, Morris HR, Easton RL, Patankast M, and Clark GF (1999). The glycobiology of gametes and fertilization. Biochim Biophys Acta 1473:196-205.

Etzioni A and Tonetti M (2000). Leukocyte adhesion deficiency II: from A to almost Z. Immunol Rev 178:138-147.

Foxall C, Watson SR, Dowbenko D, Fennie C, Lasky LA, Kiso M, Hasegawa A, Asa D, and Brandley BK (1992). The three markers of the selectin receptor family recognize a common carbohydrate epitope, the sialyl Lewis ${ }^{\times}$oligosaccharide. J Cell Biol 117:895-902.

Freeze HH (2001). Updates and perspectives on congenital disorders of glycosylation. Glycobiology 11:129R-143R.

Fukuda M (1995). Carbohydrate-dependent cell adhesion. Biorg Med Chem 3:207-215.

Fukuda M, Hiraoka N, and Yeh JC (1999). C-type lectins and sialyl Lewis ${ }^{\times}$oligosaccharides: Versatile roles in cell-cell interaction. J Cell Biol 147:467-470.

Gabius HJ (1997). Concepts of tumor lectinology. Cancer Invest 15:454-464.

Gabius HJ (2000). Biological information transfer beyond the genetic code: The sugar code. Naturwissenschaften 87:108121.

Gabius HJ (2001). Glycohistochemistry: The why and how of detection and localization of endogenous lectins. Anat Histol Embryol 30:3-31.

Gabius HJ and Bardosi A (1991). Neoglycoproteins as tools in glycohistochemistry. Prog Histochem Cytochem 22:1-66.

Gabius HJ, Gabius S, Zemlyanukhina TV, Bovin NV, Brinck U, Danguy A, Joshi SS, Kayser K, Schottelius J, Sinowatz F, Tietze LF, Vidal-Vanaclocha F, and Zanetta JP (1993). Reverse lectin histochemistry: Design and application of glycoligands for detection of cell and tissue lectins. Histol Histopathol 8:369-383.

Gabius HJ and Vehmeyer K (1988). Effect of microenvironment and cell-line type on carbohydrate-binding proteins of macrophage-like cells. Biochem Cell Biol 66:1169-1176.

Goldschmidt D, Decaestecker C, Berthe JV, Gordower L, Remmelink M, Danguy A, Pasteels JL, Salmon I, and Kiss R (1996). The contribution of image cytometry and artificial intelligence-related methods of numerical data analysis for adipose tumor histopathologic classification. Lab Invest 75: 295-306. 
Jannot MC, Kruczynski A, Limouzy A, Dangou JM, Selves J, Delsol G, Martinez J, and Kiss R (1993). Spontaneous evolution of cytoplasmic lectin binding and nuclear size and deoxyribonucleic acid content in human colorectal cancers grafted onto nude mice. Lab Invest 68:446-455.

Kansas GS (1996). Selectins and their ligands: Current concepts and controversies. Blood 88:3259-3287.

Kaji M, Ishikura H, Kishimoto T, Omi M, Ishizu A, Kimura C, Takahashi T, Kato H, and Yoshiki T (1995). E-selectin expression induced by pancreas-carcinoma-derived interleukin $1 \alpha$ results in enhanced adhesion of pancreas carcinoma cells to endothelial cells. Int J Cancer 60:712-717.

Kaltner H and Stierstorfer B (1998). Animal lectins as cell adhesion molecules. Acta Anat 161:162-179.

Kannagi R (1997). Carbohydrate-mediated cell adhesion involved in hematogenous metastasis of cancer. Glycoconjugate J 14:577-584.

Khatib AM, Kontogiannea M, Fallavollita L, Jamison B, Meterissian S, and Brodt P (1999). Rapid induction of cytokine and $\mathrm{E}$-selectin expression in the liver in response to metastatic tumor cells. Cancer Res 59:1356-1361.

Kobayashi K, Matsumoto S, Morishima T, Kawabe T, and Okamoto T (2000). Cimetidine inhibits cancer cell adhesion to endothelial cells and prevents metastasis by blocking E-selectin expression. Cancer Res 60:3978-3984.

Krause T and Turner GA (1999). Are selectins involved in metastasis? Clin Exp Metastasis 17:183-192.

Listinsky JJ, Siegal GP, and Listinsky CM (1998). $\alpha$-L-fucose: A potentially critical molecule in pathologic processes including neoplasia. Am J Clin Pathol 110:425-440.

Matsumoto S, Imaeda Y, Umemoto S, Kobayashi K, Suzuki $\mathrm{H}$, and Okamoto $\mathrm{T}$ (2002). Cimetidine increases survival of colorectal cancer patients with high levels of sialyl Lewis- $X$ and sialyl Lewis-A epitope expression on tumour cells. $\mathrm{Br} \mathrm{J}$ Cancer 86:161-167.

Muramatsu T (1993). Carbohydrate signals in metastasis and prognosis of human carcinomas. Glycobiology 3:291-296.

Nagy N, Brenner C, Markadieu N, Chaboteaux C, Camby I, Schäfer BW, Pochet R, Heizmann CW, Salmon I, Kiss R, and Decaestecker C (2001). S100A2, a putative tumor suppressor gene, regulates in vitro squamous cell carcinoma migration. Lab Invest 81:599-612.

Nakamori S, Kameyama M, Imaoka S, Furukawa H, Ishikawa $\mathrm{O}$, Sasaki Y, Kabuto T, Iwanaga T, Matsushita Y, and Irimura $T$ (1993). Increased expression of sialyl Lewis ${ }^{x}$ antigen correlates with poor survival in patients with colorectal carcinoma: Clinicopathological and immunohistochemical study. Cancer Res 53:3632-3637.

Nifant'ev NE, Tsvetkov YE, Shashkov AS, Kononov LO, Menshov VM, Tuzikov AB, and Bovin NV (1996). Selectin receptors: Synthesis of tetrasaccharides sialyl Lewis $A$ and sialyl Lewis $X$ containing a spacer group. J Carbohydr Chem 15:939-953.

Nifant'ev NE, Tsvetkov YE, Shashkov AS, Tuzikov AB, Maslennikov IV, Popova IS, and Bovin NV (1994). Receptors of selectins: Synthesis of tetrasaccharides Sia Le $\mathrm{L}^{\mathrm{a}}$ and Sia $\mathrm{Le}^{\mathrm{x}}$, and their polymer conjugates. Rus $\mathrm{J}$ Bioorgan Chem 20:311-314.
Ohyama C, Kanto S, Kato K, Nakano O, Arai Y, Kato T, Chen S, Fukuda MN, and Fukuda M (2002). Natural killer cells attack tumor cells expressing high levels of sialyl Lewis ${ }^{\times}$ oligosaccharides. Proc Natl Acad Sci USA 99:13789-13794.

Ohyama C, Tsuboi S, and Fukuda M (1999). Dual roles of sialyl Lewis ${ }^{\times}$oligosaccharides in tumor metastasis and rejection by natural killer cells. EMBO J 18:1516-1525.

Reuter G and Gabius HJ (1999). Eukaryotic glycosylation: Whim of nature or multipurpose tool? Cell Mol Life Sci 55:368-422.

Sanders WJ, Gordon EJ, Dwir O, Beck PJ, Alon R, and Kiessling RR (1999). Inhibition of L-selectin-mediated leukocyte rolling by synthetic glycoprotein mimics. J Biol Chem 274:5271-5278.

Simanek EE, McGarvey GJ, Jablonowski JA, and Wong CH (1998). Selectin-carbohydrate interactions: From natural ligands to designed mimics. Chem Rev 98:833-862.

Takada A, Ohmori K, Takahashi N, Tsuyuoka K, Yago A, Zenita K, Hasegawa A, and Kannagi R (1991). Adhesion of human cancer cells to vascular endothelium mediated by a carbohydrate antigen, sialyl Lewis A. Biochem Biophys Res Commun 179:713-719.

Tedder TF, Steeber DA, Chen A, and Engel P (1995). The selectins: Vascular adhesion molecules. FASEB J 9:866873.

Tozeren A, Kleinman HK, Grant DS, Morales D, Mercurio AM, and Byers SW (1995). E-selectin-mediated dynamic interactions of breast- and colon-cancer cells with endothelial-cell monolayers. Int J Cancer 60:426-431.

Varki A (1997). Sialic acids as ligands in recognition phenomena. FASEB J 11:248-255.

Vestweber D and Blanks JE (1999). Mechanisms that regulate the function of the selectins and their ligands. Physiol Rev 79:181-213.

Welply JK, Abbas SZ, Scudder P, Keene JL, Broschat K, Casnocha S, Gorka C, Steininger C, Howard SC, Schmuke JJ, Graneto M, Rotsaert JM, Manger ID, and Jacob GS (1994). Multivalent sialyl-LeX: Potent inhibitors of E-selectinmediated cell adhesion; reagent for staining activated endothelial cells. Glycobiology 4:259-265.

Weston BW, Hiller KM, Mayben JP, Manousos GA, Bendt KM, Liu R, and Cusack JC Jr (1999). Expression of human $\alpha$ $(1,3)$ fucosyltransferase antisense sequences inhibits selectin-mediated adhesion and liver metastasis of colon carcinoma cells. Cancer Res 59:2127-2135.

Yamazaki N, Kojima S, Bovin NV, André S, Gabius S, and Gabius HJ (2000). Endogenous lectins as targets for drug delivery. Adv Drug Deliv Rev 43:225-244.

Yoshitani N, Mori E, and Takasaki S (2001). Detection of carbohydrate recognition molecules on the plasma membrane of boat sperm by dextran-based multivalent oligosaccharide probes. Glycobiology 11:313-320.

Zhang J, Nakayama J, Ohyama C, Suzuki M, Suzuki A, Fukuda M, and Fukuda MN (2002). Sialyl Lewis X-dependent lung colonization of B16 melanoma cells through a selectinlike endothelial receptor distinct from E- and P-selectin. Cancer Res 62:4194-4198. 